\title{
Lowering of postprandial lipids in individuals with type 2 diabetes treated with alogliptin and/or pioglitazone: a randomised double-blind placebo-controlled study
}

\author{
B. Eliasson • D. Möller-Goede • K. Eeg-Olofsson • \\ C. Wilson • J. Cederholm • P. Fleck • M. Diamant • \\ M.-R. Taskinen • U. Smith
}

Received: 5 August 2011 / Accepted: 5 December 2011 / Published online: 12 January 2012

(C) Springer-Verlag 2012

\begin{abstract}
Aims/hypothesis Pharmacological augmentation of glucagon-like peptide 1 receptor signalling by dipeptidyl peptidase 4 (DPP-4) inhibition reduced intestinal lipoprotein secretion in experimental studies, suggesting that DPP-4 inhibitors may ameliorate dyslipidaemia and thus reduce cardiovascular risk in patients with type 2 diabetes. We assessed the effects of alogliptin (Alo) and Alo co-administered with pioglitazone (Pio) vs placebo (Pbo) on triacylglycerol (TG)-
\end{abstract}

B. Eliasson and D. Möller-Goede contributed equally to this study.

Electronic supplementary material The online version of this article (doi:10.1007/s00125-011-2447-3) contains peer-reviewed but unedited supplementary material, which is available to authorised users.

B. Eliasson $(\bowtie) \cdot$ K. Eeg-Olofsson $\cdot$ U. Smith

Lundberg Laboratory for Diabetes Research, Department of

Molecular and Clinical Medicine, Institute of Medicine,

University of Gothenburg, Sahlgrenska University Hospital,

41345 Göteborg, Sweden

e-mail: bjorn.eliasson@gu.se

D. Möller-Goede $\cdot$ M. Diamant

VU University Medical Center,

Diabetes Center/Department of Internal Medicine,

Amsterdam, the Netherlands

C. Wilson $\cdot$ P. Fleck

Takeda Global Research \& Development Center,

Deerfield, IL, USA

J. Cederholm

Department of Public Health and Caring Sciences/Family

Medicine and Clinical Epidemiology, Uppsala University,

Uppsala, Sweden

M.-R. Taskinen

Institute of Clinical Medicine, Division of Cardiology,

University of Helsinki, Biomedicum,

Helsinki, Finland rich lipoproteins in type 2 diabetes before and following a high-fat meal.

Methods Seventy-one patients (age 18-70 years), who did not reach $\mathrm{HbA}_{1 \mathrm{c}} 6.5 \%$ (48 mmol/mol) with lifestyle and/or metformin, sulfonylurea or glinide therapy, participated in this 16 week, double-centre (university hospitals) Pbo-controlled parallel-group study. All participants, people doing measurements or examinations, and people assessing the outcomes were blinded to group assignment. Fasting TG 1.7$5.0 \mathrm{mmol} / \mathrm{l}$ was among the entry criteria. Patients received a high-fat mixed meal before and 4 and 16 weeks after randomisation (allocation by central office) to Alo $(n=25)$, Alo/Pio $(n=22)$ or Pbo $(n=24)$. Blood was sampled at prespecified intervals, starting at $15 \mathrm{~min}$ before and ending $8 \mathrm{~h}$ after meal ingestion.

Results At week 16, Alo $(n=25)$ and Alo/Pio $(n=21)$ vs Pbo $(n=24)$ produced similar significant reductions in total postprandial TG response (incremental AUC [iAUC]; $p<0.001$ ), as well as in chylomicron TG $(p<0.001)$ and VLDL1 TG iAUCs $(p<0.001$ and $p=0.012$, respectively). Postprandial chylomicron apolipoprotein B-48 iAUC showed a significant decrease after Alo treatment $(p=0.028)$, and a non-significant trend towards a decrease with Alo/Pio $(p=0.213)$. The incidence of adverse events was low and consistent with previous studies. Conclusions/interpretation Treatment with Alo and Alo/Pio produced significant reductions in postprandial TG and TGrich lipoproteins, contributing to an improved overall cardiometabolic risk profile in type 2 diabetes. The data support the concept that incretins not only modulate glucose metabolism but also influence chylomicron metabolism in intestinal cells.

Trial registration: ClinicalTrials.gov number NCT00655863. Funding: The study was funded by Takeda Global Research \& Development. 
Keywords Alogliptin · DPP-4 inhibitor · Incretins . Postprandial lipids · Postprandial lipoproteins · Type 2 diabetes

$\begin{array}{ll}\text { Abbreviations } \\ \text { AE } & \text { Adverse event } \\ \text { Alo } & \text { Alogliptin } \\ \text { ApoB } & \text { Apolipoprotein B } \\ \text { DPP-4 } & \text { Dipeptidyl peptidase } 4 \\ \text { GIP } & \text { Glucose-dependent insulinotropic polypeptide } \\ \text { GLP-1 } & \text { Glucagon-like peptide 1 } \\ \text { HDL-C } & \text { HDL-cholesterol } \\ \text { iAUC } & \text { Incremental AUC } \\ \text { IDL } & \text { Intermediate-density lipoprotein } \\ \text { LDL-C } & \text { LDL-cholesterol } \\ \text { Pbo } & \text { Placebo } \\ \text { Pio } & \text { Pioglitazone } \\ \text { Sf } & \text { Subfraction } \\ \text { TG } & \text { Triacylglycerol } \\ \text { TRL } & \text { Triacylglycerol-rich lipoprotein } \\ \text { RLP } & \text { Remnant-like lipoprotein }\end{array}$

\section{Introduction}

As the incidence and prevalence of type 2 diabetes increases globally, different therapies are required to provide individualised and effective glucose-lowering treatment. The goals are not only to optimise glycaemic control but also to modify favourably cardiovascular risk factors. These particularly include diabetic dyslipidaemia, which is characterised by elevated levels of triacylglycerol (TG)-rich lipoproteins (TRLs) and apolipoprotein B (ApoB), low levels of HDL-cholesterol (HDL-C) and a preponderance of small atherogenic LDL particles [1]. In type 2 diabetes, the serum TG levels are frequently elevated both in the fasting and the postprandial states, indicating elevation of the highly atherogenic TRL remnants. Recent evidence also highlights the importance of TRLs as a significant risk factor for cardiovascular disease [2].

Recent data have shown that glucagon-like peptide 1 (GLP-1) plays an important role in the assembly and secretion of intestinal chylomicrons [3, 4]. Intestinal lipoprotein secretion in both mice and hamsters was reduced by potentiation of endogenous incretin action via inhibition of dipeptidyl peptidase 4 (DPP-4) - the primary enzyme involved in the in vivo degradation of at least two incretin hormones released in response to nutrient ingestion, GLP-1 and glucose-dependent insulinotropic peptide - and pharmacological augmentation of GLP-1 receptor signalling [4]. In agreement with this, the GLP-1 receptor agonist exenatide has been shown to have positive effects on postprandial lipaemia in individuals with type 2 diabetes by specifically reducing ApoB-48-containing particles [5].
So far, there is limited information on the effects of DPP-4 inhibitors on postprandial lipid and lipoprotein metabolism in patients with type 2 diabetes. In one study, 4 weeks of treatment with vildagliptin was associated with a reduction in postprandial plasma triacylglycerol and ApoB-48containing TRLs after a fat-rich meal [6]. Recently, treatment with sitagliptin for 6 weeks was shown to reduce postprandial plasma levels of TG-rich lipoproteins of both intestinal and hepatic origins [7]. Taken together, these results suggest that DPP-4 inhibitors may offer a novel approach of reducing cardiovascular risk in patients with type 2 diabetes.

Inhibition of DPP-4 activity augments glucose-stimulated insulin secretion and reduces glucagon secretion, thus lowering glycaemia. Alogliptin (Alo) is a well-tolerated oral selective inhibitor of DPP-4. Interestingly, there also seems to be an interaction between DPP-4 levels and other bloodglucose-lowering treatments, as thiazolidinediones appear to reduce DPP-4 activity [8] and may also have beneficial effects on the lipid profile [9-11]. Theoretically, such a combination of therapies may potentiate the favourable effects on both lipid and glucose metabolism in patients with type 2 diabetes.

Clinical studies of pioglitazone (Pio) have demonstrated improvements in glycaemic control as well as robust decreases in TG and increases in HDL-C [12]. Additionally, Pio treatment has also been associated with beneficial effects on postprandial TG in type 2 diabetes [9]. Thus, combined treatment with Pio and a DPP-4 inhibitor, such as Alo, may provide an advantage in terms of both treating hyperglycaemia and lowering cardiovascular risk in type 2 diabetes.

Given the potential complementary mechanisms of action of these two agents, we initiated a two-centre randomised double-blind Pbo-controlled parallel-group study to assess the effects of Alo monotherapy or Alo co-administered with Pio, compared with Pbo, on postprandial lipids and lipoprotein metabolism in individuals with type 2 diabetes. The primary objective was to evaluate the effects of the various treatment regimens on postprandial TG-rich lipoproteins. The secondary objectives of this study were to evaluate effects on BMI and measures of glycaemic control.

\section{Methods}

Patients Individuals were recruited from community hospital diabetes clinics and by newspaper advertisements. Seventyone patients were enrolled (Fig. 1). Study participants were aged between 18 and 70 years, had type 2 diabetes and had not reached adequate glycaemic control with diet and exercise or a stable dose of metformin, sulfonylurea or glinide for more than 3 months prior to screening. Entry criteria included a 
Fig. 1 Patient flow chart

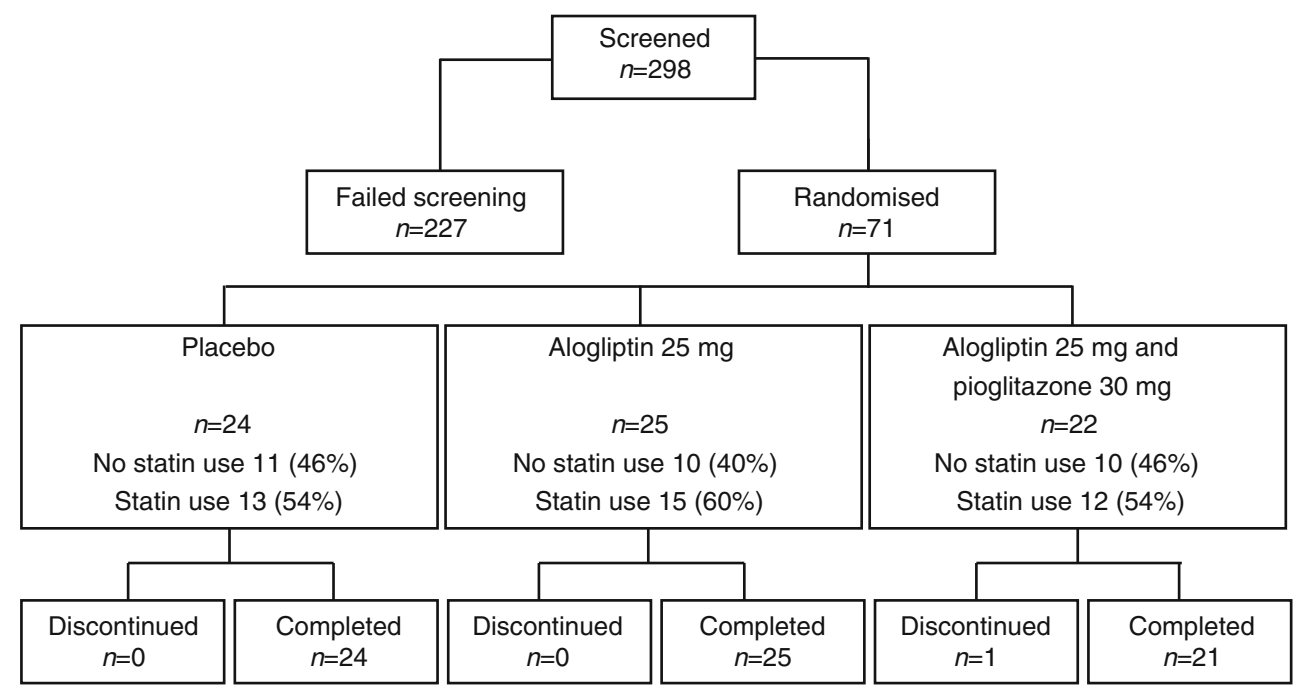

baseline $\mathrm{HbA}_{1 \mathrm{c}} 6.5-9.0 \%$ (48-75 $\left.\mathrm{mmol} / \mathrm{mol}\right)$, fasting plasma glucose $(\mathrm{FPG})<13.3 \mathrm{mmol} / \mathrm{l}$, fasting TG 1.7-5.0 mmol/1, BMI $23-45 \mathrm{~kg} / \mathrm{m}^{2}$, an ApoE3/3 or E3/4 phenotype, and stable statin and/or ezetimibe therapy or no lipid-lowering therapy within 3 months prior to screening. All other non-excluded medications should have been taken with a stable dose for at least 4 weeks prior to screening. The participants agreed to maintain their previous diet and exercise regimen during the full course of the study.

Exclusion criteria included: the use of weight-lowering drugs, corticosteroids or any investigational blood-glucoseor lipid-lowering agent (other than statins or ezetimibe) within the past 3 months; history of type 1 diabetes; history of drug or alcohol abuse within the past 2 years; systolic or diastolic blood pressure $>160 \mathrm{mmHg}$ or $>100 \mathrm{mmHg}$, respectively; alanine transaminase level of $>2.5$ times the upper limit of normal; serum creatinine level $>133 \mu \mathrm{mol} / \mathrm{l}$; fasting cholesterol $>6.5 \mathrm{mmol} / \mathrm{l}$; any coronary interventions or history of myocardial infarction within the past 6 months; or allergies to Alo or Pio. Women of childbearing potential agreed to use adequate contraception from screening throughout the duration of the study.

The study protocol was approved by the ethics review board at each study site and was in accordance with the principles of the Declaration of Helsinki. All study participants gave written informed consent before screening.

Study design This was a 16-week phase IIIB multicentre randomised double-blind Pbo-controlled parallel-group study comparing the effects of Alo alone, combination therapy with Alo and Pio, or Pbo. It was performed between August 2007 and December 2009 in two study sites (university hospitals): Sweden and the Netherlands.

Following the screening visit and confirmation of eligibility, patients were randomised (allocation by central office) within 14 days at baseline to one of three treatment groups in a 1:1:1 ratio to receive Alo $25 \mathrm{mg}$ daily, co-administration of Alo $25 \mathrm{mg}$ and Pio $30 \mathrm{mg}$ (Alo/Pio) daily, or Pbo. All participants, people doing measurements or examinations, or people assessing the outcomes were blinded to group assignment. Mean adherence during the study was similar for the Pbo (96.1\%), Alo (97.8\%) and Alo/Pio groups (97.4\%). The participants returned to the study site at weeks 4,8 and 16 for study assessments. Individuals were stratified by statin use (no therapy in the previous 3 months vs stable therapy with statin and/or ezetimibe for at least 3 months). Patients were asked to fast overnight for $8 \mathrm{~h}$ and to abstain from alcohol ingestion for 3 days and from strenuous exercise for $24 \mathrm{~h}$ prior to the screening, baseline and weeks 4,8 and 16 visits.

At baseline, week 4 and week 16 a standardised meal was given in the morning. No study medication was given before the test meal at baseline. Study medication was taken with $200 \mathrm{ml}$ water $30 \mathrm{~min}$ prior to the meal at week 4 and week 16. The standardised test meal, which contained approximately 3,845 kJ with $14 \%$ protein, $49 \%$ fat, and 36\% carbohydrates, was consumed within $10 \mathrm{~min}$. Blood samples were drawn from catheters placed in antecubital veins at 15 and 5 min before starting the meal, and $0.25,0.5,1,2,3,4,5,6,7$ and $8 \mathrm{~h}$ after the meal. Participants were allowed to drink only water until the final blood sample had been collected.

Fasting total cholesterol, HDL-C, LDL-cholesterol (LDL-C), small dense LDL and serum TG were assessed at every visit. $\mathrm{HbA}_{1 \mathrm{c}}$ was obtained at the baseline, week 8 and week 16 visits. Glucose, insulin and C-peptide samples were obtained at every time point of the standardised test meal. Total TG and the indicated lipoprotein fractions were assessed as described below in the samples obtained at $-5 \mathrm{~min}$ and 2, 3, 4, 6 and $8 \mathrm{~h}$ after the meal).

Laboratory values Plasma glucose, insulin, C-peptide and $\mathrm{HbA}_{1 \mathrm{c}}$ were analysed at the Department of Clinical Chemistry, Sahlgrenska University Hospital, Göteborg, Sweden. We used 
HOMA-IR to assess insulin resistance [13]. GLP-1 and glucagon were analysed at the Department of Biomedical Sciences, Panum Institute, University of Copenhagen, Denmark. Concentrations of lipids, lipoproteins and apolipoproteins were measured at Biomedicum, University of Helsinki, Finland. Plasma lipoprotein fractions (chylomicrons $>400$, VLDL1 subfraction [Sf] 60-400, VLDL2 Sf 20-60 and intermediatedensity lipoprotein [IDL] Sf 12-20) were separated by densitygradient ultracentrifugation as described previously [14]. TG, cholesterol, ApoB-48 and ApoB-100 concentrations were analysed in total plasma and in all fractions. TG and cholesterol were measured by automated enzymatic methods (Cobas Mira S Analyzer; Hoffman-La Roche, Basel, Switzerland) and LDL-C was calculated by the Friedewald equation [15].

Concentrations of ApoB-48 and ApoB-100 were measured in lipoprotein fractions using SDS-PAGE [16]. The bands represented by ApoB-48 and ApoB-100 were analysed with Image-Master 1D software (Amersham Pharmacia Biotech, Newcastle upon Tyne, UK). CVs for ApoB-48 and ApoB-100 were $9.65 \%$ and $5.03 \%$, respectively. The detection limit has been reported to be between 0.01 and $0.02 \mathrm{mg} / \mathrm{l}$ [16]. Plasma total ApoB-48 was analysed using an ELISA kit (AKHB48E, Shibayagi, Japan; CV 9.4\% and lower detection limit $0.4 \mathrm{ng} / \mathrm{ml}$ ) [17]. Remnant-like lipoprotein (RLP) cholesterol was quantified using an immunoseparation assay (Otsuka, Tokyo, Japan). ApoE phenotyping was performed in serum by isoelectric focusing.

Statistical analysis The primary endpoint was the change from baseline in postprandial incremental AUC (iAUC) for total plasma TG at week 16. Secondary endpoints included postprandial iAUC changes for total plasma TG at week 4 and postprandial iAUC changes for all lipid and lipoprotein variables described above at week 4 and week 16 . The primary analysis was conducted using the full analysis set and an analysis of covariance (ANCOVA) model with change from baseline in iAUC for TG as the response variable, treatment and statin use as fixed effects and baseline iAUC for TG as a covariate. Comparisons between each pair of treatment arms were derived from the primary analysis model and tested at a nominal two-sided 0.05 significance level. No multiplicity adjustments were used. Secondary variables were analysed using the full analysis set and a model similar to the primary analysis model. Missing values of primary or secondary variables were imputed using last observation carried forward (LOCF). Statistical analysis was performed using SAS version 8.2.

\section{Results}

Patient characteristics Table 1 lists the patient characteristics in the three treatment groups. The entire study population was white and $70 \%$ were male. No meaningful differences in demographic characteristics, medical history or use of concomitant medications were observed among the treatment groups at baseline. Diabetes was mostly well controlled, and only two (Pbo), five (Alo) and two patients (Alo/Pio) had $\mathrm{HbA}_{1 \mathrm{c}}$ values $>7.5 \%(58 \mathrm{mmol} / \mathrm{mol})$. Only one participant (in the Pbo group) was treated with ezetimibe, while no patient used oestrogen or progestin agents.

Metabolic and lipid variables Electronic supplementary material (ESM) Table 1 reports the between-group comparisons of fasting lipids and lipoproteins, including plasma ApoB-48 and RLP cholesterol. Treatment with Alo for 16 weeks reduced fasting TG by $0.56 \mathrm{mmol} / \mathrm{l}(p=0.003)$ and Alo/Pio lowered fasting TG by $0.81 \mathrm{mmol} / \mathrm{l}(p<0.001)$ compared with Pbo. Levels of ApoB-48 as well as RLP cholesterol were significantly reduced by both Alo and Alo/Pio treatment compared with Pbo. Treatment with Alo/Pio, but not Alo alone, significantly increased fasting HDL-C $(0.23 \mathrm{mmol} / 1$ from baseline $)$ compared with a decrease of $0.05 \mathrm{mmol} / \mathrm{l}$ for Pbo $(p<0.001)$. Fasting total cholesterol and LDL-C were not changed in any treatment group at week 16. Alo/Pio slightly reduced fasting NEFA levels compared with PLO $(-0.10 \mathrm{mmol} / 1, p=0.028)$, while there was no effect of Alo treatment $(-0.02 \mathrm{mmol} / 1, p=0.68)$. After 16 weeks, NEFA levels showed a non-significant trend to be lower after Alo/Pio than Alo treatment $(-0.09 \mathrm{mmol} / \mathrm{l}$, $p=0.066$ ).

Figure $2 \mathrm{a}-\mathrm{c}$ shows baseline and endpoint concentrations of total TG, chylomicron TG and VLDL1 TG in the three different treatment arms. Similarly, Fig. $2 \mathrm{~d}-\mathrm{f}$ gives total plasma ApoB-48, chylomicron ApoB-48, and VLDL1 ApoB-48 in the three treatment arms. Table 2 reports the between-group comparisons of postprandial iAUC (0-8) of lipid and lipoprotein variables. Overall, the effects of Alo and Alo/Pio treatments on lipids and ApoB-48 changes in different TRL species were seen already at 4 weeks (data not shown) and were maintained over the 16 week treatment period.

Both Alo and Alo/Pio treatment produced similar and statistically significant $(p<0.001)$ reductions at week 16 in total postprandial TG (iAUC) compared with Pbo. Although individuals in the Alo group exhibited a trend to greater mean reduction compared with the Alo/Pio group ( -3.47 vs $-2.87 \mathrm{mmol} / \mathrm{l} \times \mathrm{h}$, respectively), this difference was not statistically significant $(p=0.445)$. When sex was included as a covariate in the calculation the results remained virtually unchanged (data not shown).

Similarly, both Alo and Alo/Pio led to statistically significant reductions in postprandial chylomicron TG and VLDL1 TG compared with Pbo and there were no statistically significant differences between the two treatment arms. However, the numerically higher mean reduction of 
Table 1 Patient characteristics

\begin{tabular}{llll}
\hline Characteristic & Pbo $(n=24)$ & Alo $(n=25)$ & Alo/Pio $(n=22)$ \\
\hline Sex, $n(\%)$ & & & \\
Men & $20(83)$ & $15(60)$ & $15(68)$ \\
Women & $4(17)$ & $10(40)$ & $7(32)$ \\
Age (years) & $59.1(6.2)$ & $58.7(6.5)$ & $59.1(6.9)$ \\
Weight (kg) & $101.8(12.0)$ & $93.7(10.8)$ & $93.1(13.2)$ \\
Height (cm) & $178(7)$ & $174(8)$ & $173(11)$ \\
BMI (kg/m $\left.{ }^{2}\right)$ & $32.1(4.0)$ & $31.1(4.2)$ & $31.2(3.5)$ \\
Diabetes duration (years) & $5.5(3.2)$ & $6.4(3.6)$ & $5.0(3.8)$ \\
HbA 1 (\%) & $6.6(0.7)$ & $6.8(0.8)$ & $6.6(0.6)$ \\
HbA 1 (mmol/mol) & $49(8)$ & $51(9)$ & $49(7)$ \\
FPG (mmol/l) & $8.9(1.6)$ & $9.3(2.8)$ & $116.5(2.0)$ \\
Fasting plasma & $126.4(63.9)$ & $116.0(53.5)$ & $6.7(5.4)$ \\
insulin (pmol/1) & & & \\
HOMA-IR (\%) & $7.1(3.5)$ & $6.9(3.6)$ & $21(95)$ \\
Concomitant medications, $n(\%)$ & & & $12(55)$ \\
Metformin/other OHA & $23(96)$ & $22(88)$ & $9(41)$ \\
Lipid-lowering agents & $13(54)$ & $15(60)$ & $7(32)$ \\
RAS blockers & $13(54)$ & $9(36)$ & $6(27)$ \\
Beta-blocking agents & $8(33)$ & $7(28)$ & $4(18)$ \\
Diuretics & $5(21)$ & $6(24)$ &
\end{tabular}

Data are means (SD) or proportions

OHA, oral glucose-lowering agents; RAS, renin-angiotensin system by $0.7 \mathrm{mmol} / 1$, while it decreased by $0.9 \mathrm{mmol} / 1$ and $2.1 \mathrm{mmol} / \mathrm{l}$ in patients randomised to Alo and Alo/Pio, respectively. HOMA-IR was similar at baseline across the different treatment groups and did not change from baseline in patients treated with Pbo and Alo. However, HOMA-IR decreased from baseline by a statistically significant 3.06 ( $p=0.005$ vs Alo) in patients randomised to Alo/Pio (Fig. $3 \mathrm{c}$ ). There were no significant differences in postprandial NEFA levels between the treatment groups (Pbo vs Alo or Alo/Pio, or Alo vs Alo/Pio) after 16 weeks of treatment.

Active GLP-1 and glucagon levels during the meal test at week 16 are presented in Fig. 4a,b. Postprandial levels of active GLP-1 were significantly increased in patients treated with Alo/Pio compared with Pbo, but the largest increase was seen in patients treated with Alo only. Postprandial glucagon levels were suppressed to a similar and statistically significant degree in patients treated with Alo and Alo/Pio compared with Pbo.

Safety The incidence of adverse events (AEs) ranged from $59 \%$ for the Alo/Pio group to $76 \%$ for the Alo group. More events were considered by the investigator to be related to study medication in the Alo/Pio group than the other two treatment groups. The majority of events (71/81) were considered mild in severity, with seven considered moderate and three severe. More individuals in the Alo and Alo/Pio (five and three, respectively) experienced nasopharyngitis than in the $\mathrm{Pbo}$ group (one). Hypoglycaemia was reported for three individuals in the Alo/Pio group but none in the other groups. Anaemia respectively. In patients randomised to $\mathrm{Pbo}, \mathrm{FPG}$ increased 

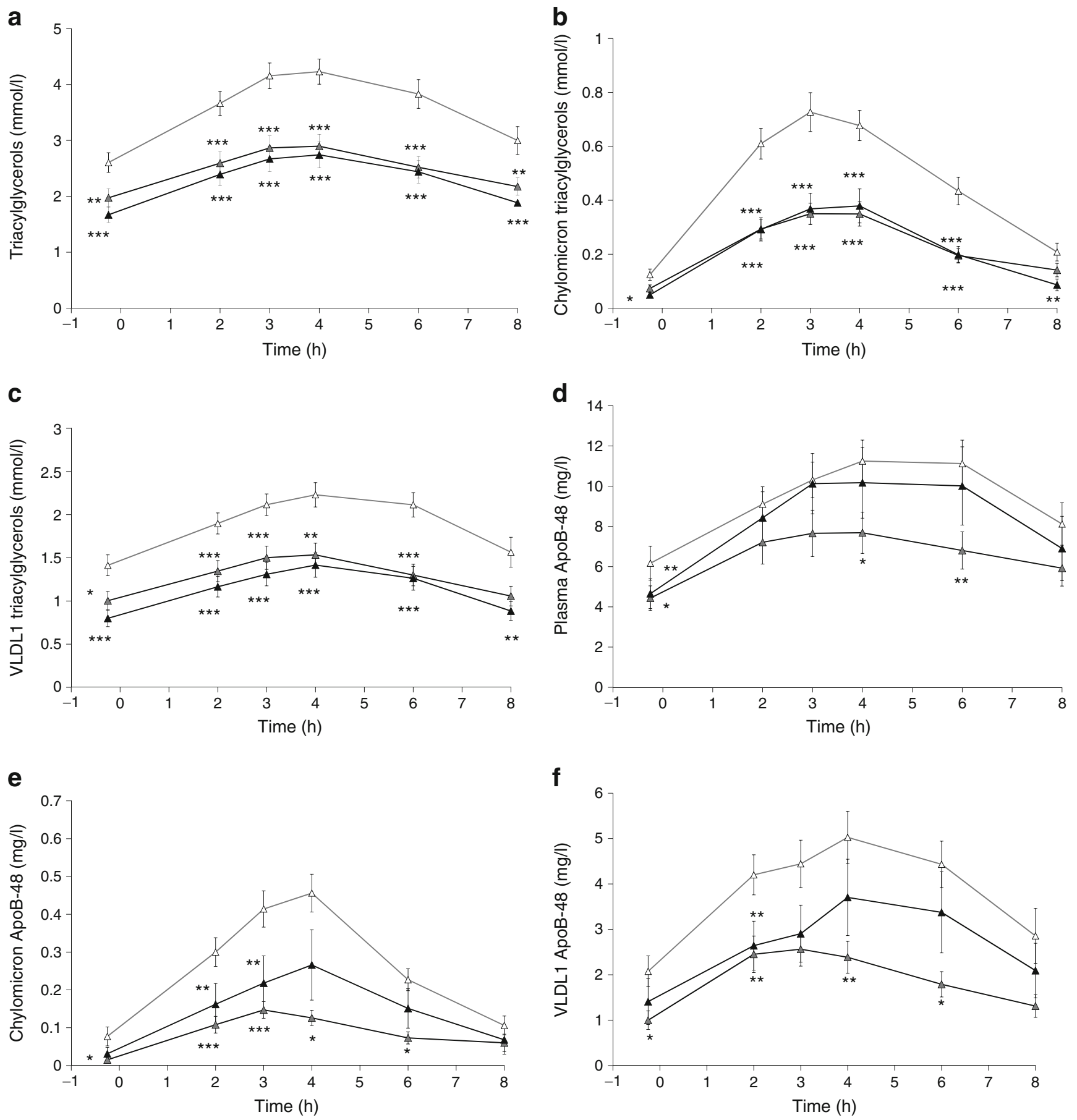

Fig. 2 Mean ( \pm SE) postprandial TG and ApoB-48 at week 16. a Total TG; (b) chylomicron TG; (c) VLDL1 TG; (d) ApoB-48; (e) chylomicron ApoB-48; and (f) VLDL1 ApoB-48. Concentrations of ApoB-48 were measured in lipoprotein fractions using SDS-PAGE. $p$ values for

was reported for three individuals in the Alo group vs one in the Pbo group and none in the Alo/Pio group. Two cases of anaemia in the Alo and the one case in the Pbo group were ongoing at the time of study completion. Of the severe AEs reported during the study, three were reported for one person in the Alo/Pio group as a result of one incident (fall, head injury, the least squares mean treatment difference: ${ }^{*} p<0.05$, $* * p<0.01$ and $* * * p<0.001$ compared with Pbo. White triangles, Pbo; grey triangles, Alo; black triangles, Alo/Pio

and concussion), and were considered by the investigator to be not related to study drug. The other events were gastric ulcer and nephrolithiasis, reported for one participant each in the Alo group. Both were assessed as related to study drug. No clinically relevant changes in laboratory tests, ECGs or physical examination findings were observed. Alo was generally safe 


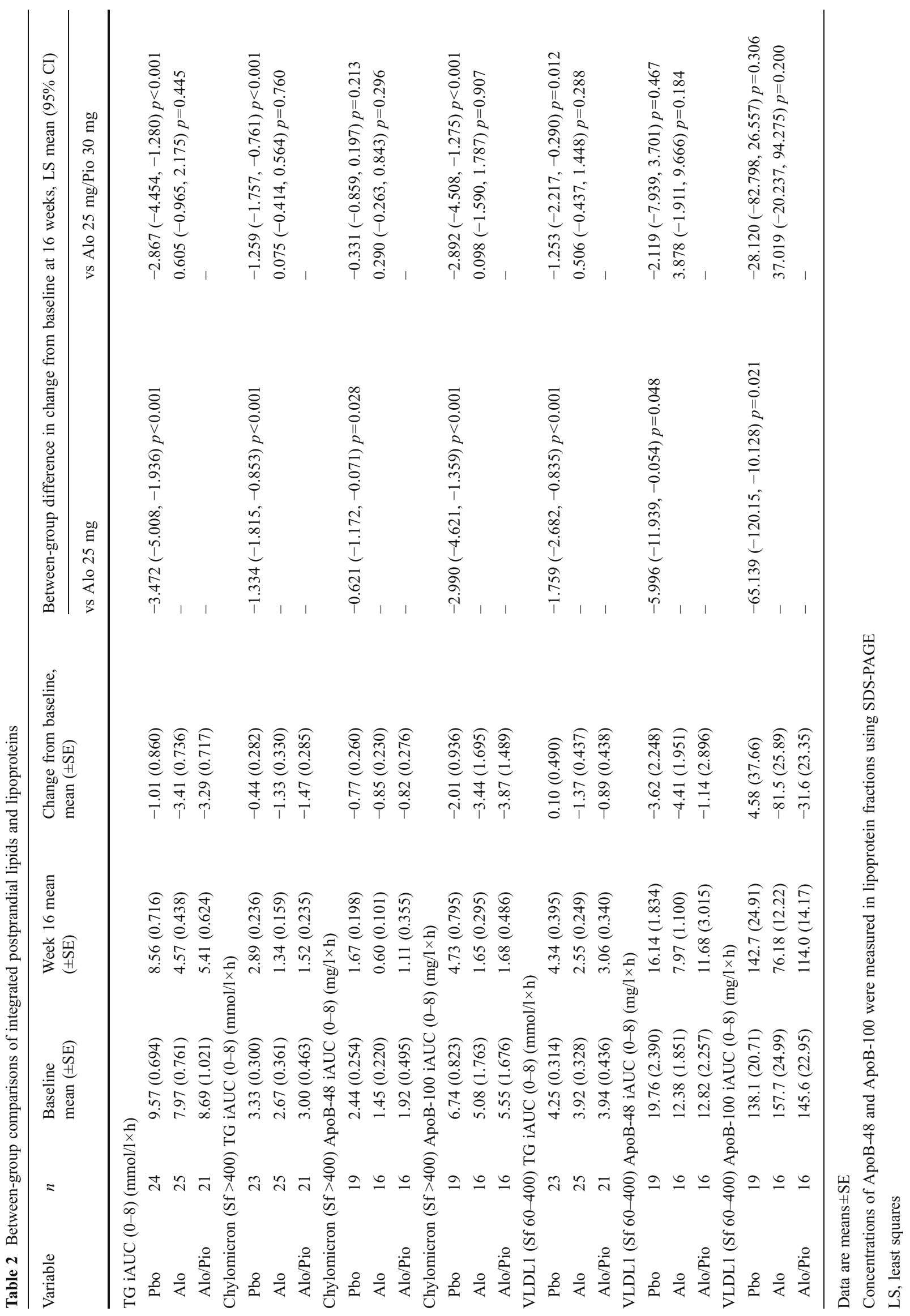



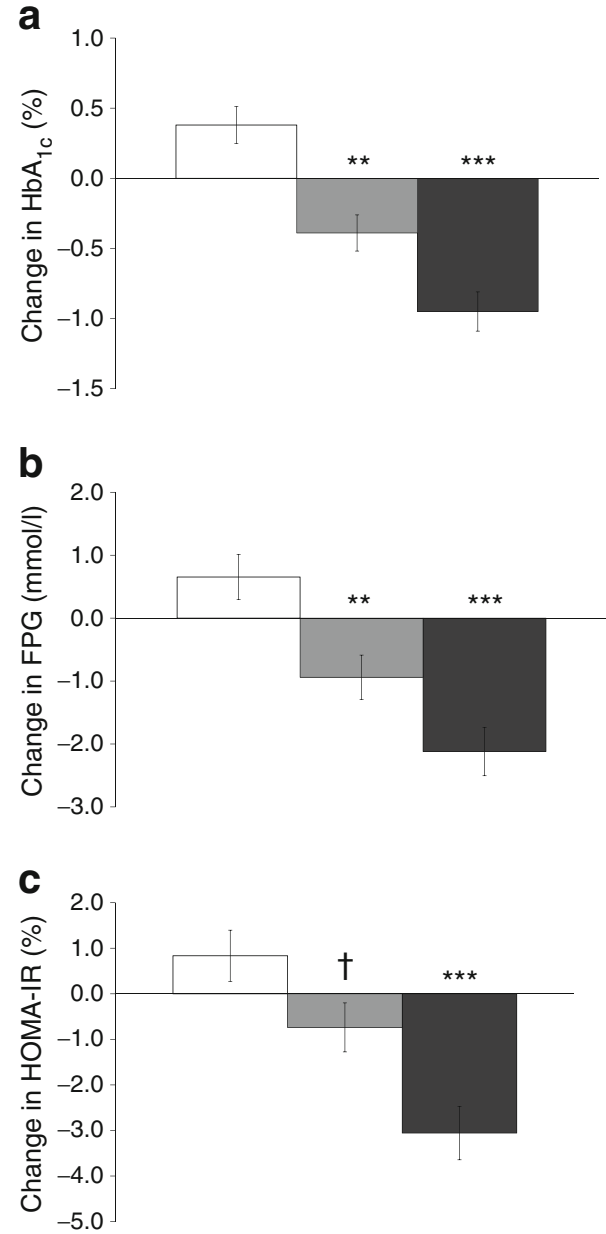

Fig. 3 Mean $( \pm \mathrm{SE})$ change from baseline to week 16 in: (a) $\mathrm{HbA}_{1 \mathrm{c}}$, baseline values Pbo $6.6 \%$, Alo 6.8\% and Alo/Pio 6.6\% (to convert values for $\mathrm{HbA}_{1 \mathrm{c}}$ in $\%$ into $\mathrm{mmol} / \mathrm{mol}$, subtract 2.15 and multiply by 10.929); (b) FPG, baseline values Pbo $8.9 \mathrm{mmol} / \mathrm{l}$, Alo $9.3 \mathrm{mmol} / \mathrm{l}$ and Alo/Pio $8.5 \mathrm{mmol} / \mathrm{l}$; and (c) HOMA-IR, baseline values Pbo $7.1 \%$, Alo $6.9 \%$ and Alo/Pio 6.7\%. $p$ values for the least squares mean treatment difference: ${ }^{\dagger} p=0.048,{ }^{* *} p<0.01$ and $* * * p<0.001$ compared with Pbo. White bars, Pbo; grey bars, Alo; dark grey bars, Alo/Pio

a

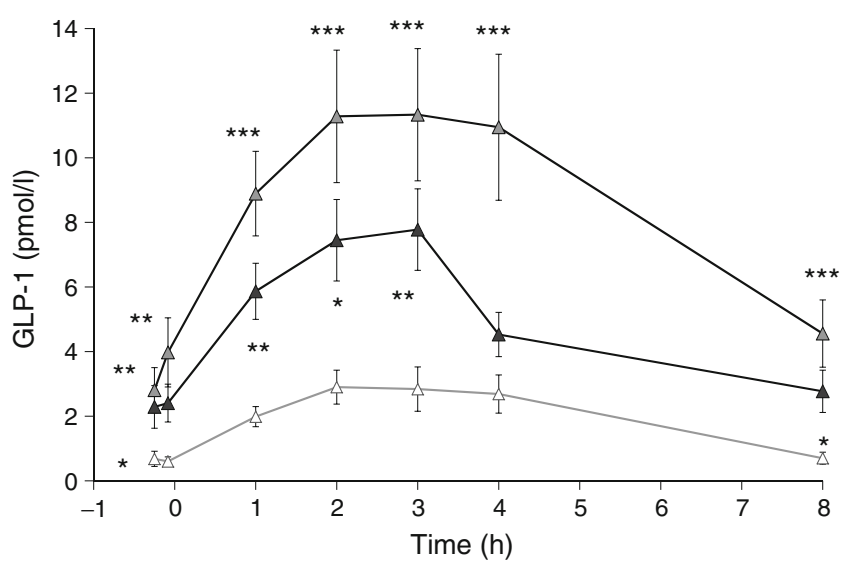

and well tolerated in this study as monotherapy and as coadministered with Pio. The incidence of AEs and severe AEs was low and consistent with previous studies.

\section{Discussion}

The major finding of this randomised double-blind Pbocontrolled study is that the DPP-4 inhibitor Alo at a dose of $25 \mathrm{mg}$ daily for 16 weeks caused highly significant reductions in postprandial total TG and chylomicron TG following a fat-rich meal, and also significantly reduced fasting TG and RLP cholesterol levels in patients with type 2 diabetes. Alo treatment significantly decreased ApoB-48 in chylomicrons, indicating that the number of chylomicron particles was reduced. The finding that the combination of Alo with Pio did not further reduce postprandial TG response or fasting TG is surprising, although it significantly increased fasting HDL-C compared with Alo alone. As expected, treatment with Alo/Pio decreased $\mathrm{HbA}_{1 \mathrm{c}}$ to a significantly greater extent than Alo alone. Moreover, GLP-1 and glucosedependent insulinotropic polypeptide (GIP) levels were increased but glucagon levels reduced in response to an oral fat load in both study arms. These results show that treatment with Alo improved the overall cardiometabolic risk profile in patients with type 2 diabetes; results were augmented by the Alo/Pio combination.

Our data expand results from a previous pilot study using vildagliptin for 4 weeks and from a recent study where sitagliptin treatment for 6 weeks was reported to reduce postprandial elevations of TG and ApoB-48 in response to a fat-rich meal in type 2 diabetes $[6,7]$. Likewise, vildagliptin reduced chylomicron ApoB-48 levels, and exenatide reduced postprandial lipaemia with a robust reduction for chylomicron ApoB-48 [5, 18]. Taken together, these data suggest that modulation of the GLP-1 system not only

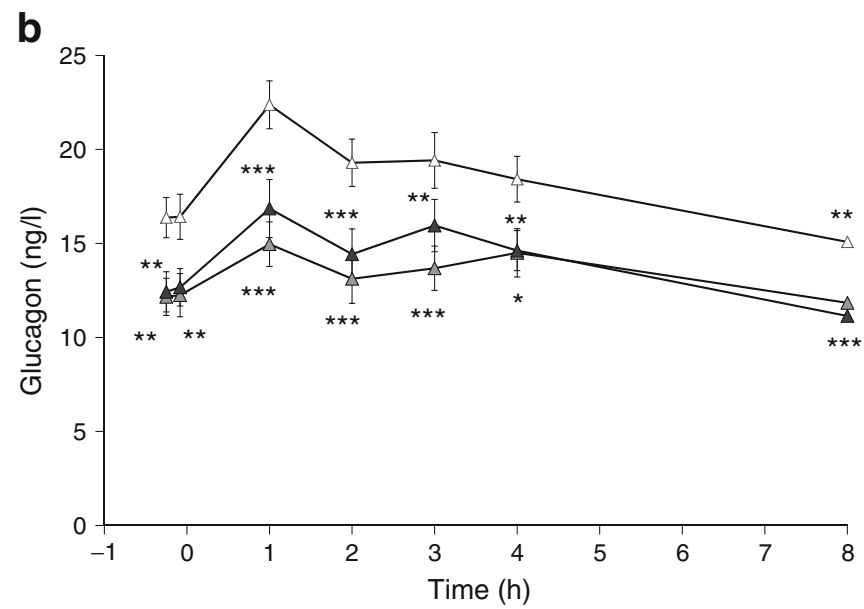

Fig. 4 Mean $\left( \pm\right.$ SE) postprandial active GLP-1 (a) and glucagon (b) at week 16. $p$ values for the least squares mean treatment difference: ${ }^{*} p<0.05$, ${ }^{* *} p<0.01,{ }^{* * *} p<0.001$ compared with Pbo. White triangles, Pbo; grey triangles, Alo; black triangles, Alo/Pio 
influences glucose metabolism but also favourably interferes with intestinal lipid metabolism $[3,4,19]$. The data also suggest that the effect of DDP-4 inhibitors seems to be a class effect, but there seems to be some differences in the efficacy of DPP-4 inhibitors to modulate lipid levels. In contrast to vildagliptin and sitaglipitin, Alo reduced the fasting TG levels as well as the fasting RLP cholesterol levels. Whether this is due to specific characteristics of Alo as a DPP-4 inhibitor and/or an increased potency is currently unclear.

Postprandial TRLs comprise, in addition to chylomicrons ( $\mathrm{Sf}>400$ ) containing ApoB-48, large VLDL particles (VLDL1 Sf 60-400) containing both ApoB-48 and ApoB100, smaller VLDL particles (VLDL2 Sf 20-60) and IDL particles containing mainly ApoB-100, all contributing to the elevation of serum TG. We report here significant decreases in levels of the major chylomicron components (ApoB-48, TG and cholesterol) in individuals treated with Alo. Interestingly, sitaglipitin has been demonstrated to decrease postprandial TRL TG, cholesterol and ApoB-48 in fructose-fed rodents [4], and it has been suggested that sitagliptin reduces intestinal postprandial TG and cholesterol secretion. Exendin-4 caused similar changes in lipids and ApoB-48 in the TRL fraction, as did sitagliptin in hamsters. In contrast, the GLP-1 receptor antagonist exendin (9-35) blocked the effect of exendin- 4 on the TRL response to an oral fat load. The authors also provided evidence that the effects of sitagliptin could not be explained by the action on gastric emptying or via GIP receptor signalling. In summary, this elegant study demonstrated that GLP-1 regulates the assembly and/or secretion of chylomicrons by directly influencing enterocyte ApoB-48 secretion ex vivo.

It should be recognised that large VLDL1 particles contain ApoB-48 remnants as well as ApoB-100 particles of hepatic origin and their remnants. We report here for the first time that Alo significantly also reduced VLDL1 ApoB-48 iAUC and TG iAUC, suggesting that chylomicron remnants in the VLDL1 fraction were decreased. The lack of significant decrease of the VLDL/IDL fraction by vildagliptin may have been due to a power problem or to the fact that large VLDL1 species, which contain the major part of chylomicron remnants, were not specifically isolated [6]. This was the case in the study by Tremblay et al. [7]. It was recently reported that RLP TG levels correlate closely with total postprandial TG levels. Notably, RLP TG constitutes about $80 \%$ of the total postprandial TG concentration at 3-6 h after an oral fat load [20]. Thus, the total burden of remnant particles seems to be considerably more than previously reported.

In addition to the postprandial reduction of VLDL1 ApoB-48, VLDL1 ApoB-100 and TG iAUC were significantly reduced by Alo. This may be due to either improved clearance or reduced production rate of large VLDL particles.
As chylomicrons and VLDL particles and their remnants compete for the same catabolic pathways, both at the first step of lipolysis by LPL and at the second step of uptake by liver remnant receptors [21, 22], a reduced secretion of ApoB-48 particles by itself could improve the catabolic rate of VLDL1 particles by releasing the competition for the clearance pathway. The key regulators of VLDL assembly in the liver are NEFA flux from the systemic NEFA pool, dietary fat intake and de novo lipogenesis [23-25]. Likewise, NEFA flux seems to regulate chylomicron production by intestinal cells [26], and insulin acutely suppresses ApoB-48 production due to the reduced NEFA flux [27, 28]. In the present study, postprandial NEFA levels were similarly suppressed in the three treatment groups. Thus, changes in NEFA flux are not likely to be the major mechanism explaining the improved postprandial lipaemia by Alo and by Pio/Alo. Recently, GLP-1 receptor production was demonstrated in human hepatocytes and exendin4 was shown to reduce hepatic steatosis in the absence of insulin [29]. This potential direct effect of GLP-1 on lipid metabolism offers an explanation also for the observed reduction of VLDL1 ApoB and TG in this study. Furthermore, a recent report on GLP-1 treatment in mice fed a high-fat diet showed reduced VLDL production during hyperinsulinaemic clamps without affecting lipolysis [30]. Therefore it is necessary to confirm the effect of DDP-4 inhibitors and exenatide on the kinetics of TG, ApoB-48 and ApoB-100 in the postprandial state, using stable isotopes concomitantly with quantification of liver fat, and thus to determine if the production or catabolic rate of ApoB-48 and ApoB-100 particles is modified. This would demonstrate how modulation of the GLP-1 system influences lipoprotein metabolism in humans.

An unexpected finding was that the combination of Alo/ Pio did not further decrease the postprandial TRL lipid response to the oral fat load as Pio alone decreases not only fasting TG but also postprandial lipaemia [9-11]. Recent evidence also indicates that liver fat content is reduced by Pio [31]. As liver fat content is a driving force for VLDL1 overproduction in patients with type 2 diabetes [32], one would expect to see a further reduction of VLDL1 ApoB100 and TG in the combination treatment arm. The design of the present study does not allow elucidation of a potential mechanism, which remains an open question. In contrast to the postprandial lipid response, we observed robust additive effects of Alo/Pio treatment, as compared with Alo alone, on $\mathrm{HbA}_{1 \mathrm{c}}$, FPG and HOMA-IR index. This is expected, recognising the complementary mechanisms of action of Alo stimulating insulin secretion and Pio enhancing insulin sensitivity. This agrees with data from $d b / d b$ mice, in which Alo in combination with Pio enhanced the effect on glycaemic control [33]. Thus, the lack of additive effect by Alo/Pio on the postprandial lipids argues against the possibility that the observed changes are secondary to significant ameliorations of both glycaemic control and insulin resistance by Pio. 
Together, the data support the concept that incretins not only modulate glucose metabolism, but also lipid metabolism at the intestinal and hepatic levels via GLP-1 receptor activation by correcting dysfunction of the gut-liver axis in type 2 diabetes. The exact molecular mechanisms remain to be resolved but the current study underlines the concept that DPP-4 inhibitors and GLP-1 exert several other important actions on cardiovascular risk in type 2 diabetes, in addition to improving the glycaemic control.

Funding Takeda Global Research \& Development sponsored this study. The sponsors were involved in the study design, protocol development, and the collection, review and analysis of the data.

Duality of interest CW and PF are employed by Takeda. BE and US has received speakers' fees from Takeda. MRT is a shareholder, and has received research grants from Eli Lilly, MSD and Takeda and honoraria from Novartis and Boehringer Mannheim, and speakers' fees.

Contribution statement $\mathrm{BE}, \mathrm{DMG}, \mathrm{MD}, \mathrm{KEO}, \mathrm{JC}, \mathrm{MRT}, \mathrm{US}, \mathrm{CW}$ and $\mathrm{PF}$ participated in conception and design of the study, BE, DMG, MRT, MD, JC, US, CW and JC analysed and interpreted data; BE, DMG, MRT and US drafted the article while MD, KEO, JC, CW and PF revised it critically for important intellectual content; and all authors approved the final version of the manuscript to be published.

\section{References}

1. Taskinen MR (2003) Diabetic dyslipidaemia: from basic research to clinical practice. Diabetologia 46:733-749

2. Chapman MJ, Ginsberg HN, Amarenco P et al (2011) Triglyceride-rich lipoproteins and high-density lipoprotein cholesterol in patients at high risk of cardiovascular disease: evidence and guidance for management. Eur Heart J 11:1345-1361

3. Bandsma RH, Lewis GF (2010) Newly appreciated therapeutic effect of GLP-1 receptor agonists: reduction in postprandial lipemia. Atherosclerosis 212:40-41

4. Hsieh J, Longuet C, Baker CL et al (2010) The glucagon-like peptide 1 receptor is essential for postprandial lipoprotein synthesis and secretion in hamsters and mice. Diabetologia 53:552-561

5. Bunck MC, Corner A, Eliasson B et al (2010) One-year treatment with exenatide vs. insulin glargine: effects on postprandial glycemia, lipid profiles, and oxidative stress. Atherosclerosis 212:223229

6. Matikainen N, Manttari S, Schweizer A et al (2006) Vildagliptin therapy reduces postprandial intestinal triglyceride-rich lipoprotein particles in patients with type 2 diabetes. Diabetologia 49:2049 2057

7. Tremblay AJ, Lamarche B, Deacon CF, Weisnagel SJ, Couture P (2011) Effect of sitagliptin therapy on postprandial lipoprotein levels in patients with type 2 diabetes. Diabetes Obes Metabol 13:366-373

8. Lenhard JM, Croom DK, Minnick DT (2004) Reduced serum dipeptidyl peptidase-IV after metformin and pioglitazone treatments. Biochem Biophys Res Commun 324:92-97
9. Al Majali K, Cooper MB, Staels B, Luc G, Taskinen MR, Betteridge DJ (2006) The effect of sensitisation to insulin with pioglitazone on fasting and postprandial lipid metabolism, lipoprotein modification by lipases, and lipid transfer activities in type 2 diabetic patients. Diabetologia 49:527-537

10. Bajaj M, Suraamornkul S, Hardies LJ, Glass L, Musi N, DeFronzo RA (2007) Effects of peroxisome proliferator-activated receptor (PPAR)-alpha and PPAR-gamma agonists on glucose and lipid metabolism in patients with type 2 diabetes mellitus. Diabetologia 50:1723-1731

11. Goldberg RB, Kendall DM, Deeg MA et al (2005) A comparison of lipid and glycemic effects of pioglitazone and rosiglitazone in patients with type 2 diabetes and dyslipidemia. Diabetes Care 28:1547-1554

12. Rosenblatt S, Miskin B, Glazer NB, Prince MJ, Robertson KE (2001) The impact of pioglitazone on glycemic control and atherogenic dyslipidemia in patients with type 2 diabetes mellitus. Coron Artery Dis 12:413-423

13. Matthews DR, Hosker JP, Rudenski AS, Naylor BA, Treacher DF, Turner RC (1985) Homeostasis model assessment: insulin resistance and beta-cell function from fasting plasma glucose and insulin concentrations in man. Diabetologia 28:412-419

14. Mero N, Syvanne M, Eliasson B, Smith U, Taskinen MR (1997) Postprandial elevation of apoB-48-containing triglyceride-rich particles and retinyl esters in normolipemic males who smoke. Arterioscler Thromb Vasc Biol 17:2096-2102

15. Friedewald WT, Levy RI, Fredrickson DS (1972) Estimation of the concentration of low-density lipoprotein cholesteroi in plasma, without use of the preparative ultracentrifuge. Clin Chem 18:499-502

16. Karpe F, Hamsten A (1994) Determination of apolipoproteins B48 and B-100 in triglyceride-rich lipoproteins by analytical SDSPAGE. J Lipid Res 35:1311-1317

17. Kinoshita M, Kojima M, Matsushima T, Teramoto T (2005) Determination of apolipoprotein B-48 in serum by a sandwich ELISA. Clin Chim Acta 351:115-120

18. Schwartz EA, Koska J, Mullin MP, Syoufi I, Schwenke DC, Reaven PD (2010) Exenatide suppresses postprandial elevations in lipids and lipoproteins in individuals with impaired glucose tolerance and recent onset type 2 diabetes mellitus. Atherosclerosis 212:217-222

19. Ban K, Hui S, Drucker DJ, Husain M (2009) Cardiovascular consequences of drugs used for the treatment of diabetes: potential promise of incretin-based therapies. J Am Soc Hypertens 3:245259

20. Nakajima K, Nakano T, Moon HD et al (2009) The correlation between TG vs remnant lipoproteins in the fasting and postprandial plasma of 23 volunteers. Clin Chim Acta 404:124-127

21. Dallinga-Thie GM, Franssen R, Mooij HL et al (2010) The metabolism of triglyceride-rich lipoproteins revisited: new players, new insight. Atherosclerosis 211:1-8

22. Williams KJ, Chen K (2010) Recent insights into factors affecting remnant lipoprotein uptake. Curr Opin Lipidol 21:218-228

23. Adiels M, Olofsson SO, Taskinen MR, Boren J (2008) Overproduction of very low-density lipoproteins is the hallmark of the dyslipidemia in the metabolic syndrome. Arterioscler Thromb Vasc Biol 28:1225-1236

24. Fabbrini E, Sullivan S, Klein S (2010) Obesity and nonalcoholic fatty liver disease: biochemical, metabolic, and clinical implications. Hepatology 51:679-689

25. Lewis GF, Uffelman KD, Szeto LW, Weller B, Steiner G (1995) Interaction between free fatty acids and insulin in the acute control of very low density lipoprotein production in humans. J Clin Invest 95:158-166

26. Duez H, Lamarche B, Valero R et al (2008) Both intestinal and hepatic lipoprotein production are stimulated by an acute elevation of plasma free fatty acids in humans. Circulation 117:2369-2376 
27. Adeli K, Lewis GF (2008) Intestinal lipoprotein overproduction in insulin-resistant states. Curr Opin Lipidol 19:221-228

28. Pavlic M, Xiao C, Szeto L, Patterson BW, Lewis GF (2010) Insulin acutely inhibits intestinal lipoprotein secretion in humans in part by suppressing plasma free fatty acids. Diabetes $59: 580-587$

29. Gupta NA, Mells J, Dunham RM et al (2010) Glucagon-like peptide-1 receptor is present on human hepatocytes and has a direct role in decreasing hepatic steatosis in vitro by modulating elements of the insulin signaling pathway. Hepatology 51:15841592

30. Parlevliet ET, de Leeuw van Weenen JE, Romijn JA, Pijl H (2010) GLP-1 treatment reduces endogenous insulin resistance via activation of central GLP-1 receptors in mice fed a high-fat diet. Am J Physiol Endocrinol Metab 299:E318-324

31. Belfort R, Harrison SA, Brown K et al (2006) A placebocontrolled trial of pioglitazone in subjects with nonalcoholic steatohepatitis. N Engl J Med 355:2297-2307

32. Adiels M, Taskinen MR, Packard C et al (2006) Overproduction of large VLDL particles is driven by increased liver fat content in man. Diabetologia 49:755-765

33. Moritoh Y, Takeuchi K, Asakawa T, Kataoka O, Odaka H (2009) Combining a dipeptidyl peptidase-4 inhibitor, alogliptin, with pioglitazone improves glycaemic control, lipid profiles and beta-cell function in $\mathrm{db} / \mathrm{db}$ mice. Br J Pharmacol 157:415-426 